\title{
HSV-1 Scaffolding Protein Bubbles Readily in the Absence or Presence of DNA, Allowing its Localization in Immature and Mature Nucleocapsids
}

\author{
Dennis C. Winkler, William W. Newcomb, Anastasia Aksyuk, Weimin Wu, Naiqian Cheng, and \\ Alasdair C. Steven
}

Laboratory of Structural Biology Research, National Institute of Arthritis and Musculoskeletal and Skin Diseases, National Institutes of Health, Bethesda, MD 10892 USA.

The herpes simplex virus 1 (HSV-1) capsid is an immense macromolecular assembly, $1250 \AA$ in diameter and composed of thousands of protein subunits of six different kinds. Their cumulative mass amounts to $\sim 200 \mathrm{MDa}$ and the capsid houses a $100 \mathrm{MDa}$ genome. The assembly pathway has much in common with those of tailed bacteriophages, with which HSV shares a (distant) evolutionary relationship [1]. It starts with formation of a procapsid which matures via a major structural transformation, preceded by proteolysis and coupled to DNA packaging. A-capsids are mature but empty; B-capsids are mature but retain a shrunken scaffolding shell; C-capsids are filled with DNA and have ostensibly expelled the scaffolding shell - Fig. 1 [1]. In this study we are applying "bubblegram imaging" $[2,3]$ to the various kinds of HSV capsids. Among other goals, we aim to determine whether there are any proteins present in the DNA inside the C-capsid, as there are in many bacteriophages.

Capsids were produced and isolated as previously described [4]. Cryo-EM was performed on a CM200FEG electron microscope operated at $120 \mathrm{kV}$, recording "dose series" of micrographs with 1-second exposures recorded 5 seconds apart. Each exposure imparted 15 electrons $/ \AA^{2}$.

A field of purified procapsids is shown in Fig. 2 for the first exposure (left) and the ninth exposure (right). In the latter panel, the particles show extensive bubbling in their inner (scaffolding) shell, whereas the outer shell (capsid) shows only blurring, not bubbling. These observations indicate that the scaffolding protein preUL26.5 is particularly bubbling-prone. They also demonstrate that DNA is not needed to elicit bubbling. A corresponding image pair is shown in Fig. 3 for a mixed population of Acapsids, B-capsids, and C-capsids. The A-capsids blur but show no bubbling. The B-capsids bubble internally, confirming that their internal density consists mainly if not exclusively of the scaffolding protein UL26.5 processed by removal of a C-terminal peptide [1]. In contrast, C-capsids show small bubbles distributed throughout their interior. A plausible (but not the only) candidate is residual scaffolding protein. Originally there are $\sim 1800$ copies per procapsid and, although the large majority are expelled during DNA packaging, there may be enough left to generate the observed bubbles. In this scenario, these proteins are no longer organized in a spherical shell but are re-distributed throughout the C-capsid interior.

\section{References}

[1] G. Cardone et al in "Viral Molecular Machines", eds. M.G. Rossmann and V.B. Rao (Springer, New York) p. 423.

[2] W. Wu et al, Science 335 (2012) p. 182.

[3] N. Cheng et al, J Struct. Biol. 185 (2014) p. 250.

[4] F. Booy et al, Cell 8 (1991) p. 1007.

[5] This research was supported by the Intramural Research Program of the National Institute of Arthritis and Musculoskeletal and Skin Diseases of the National Institutes of Health. 

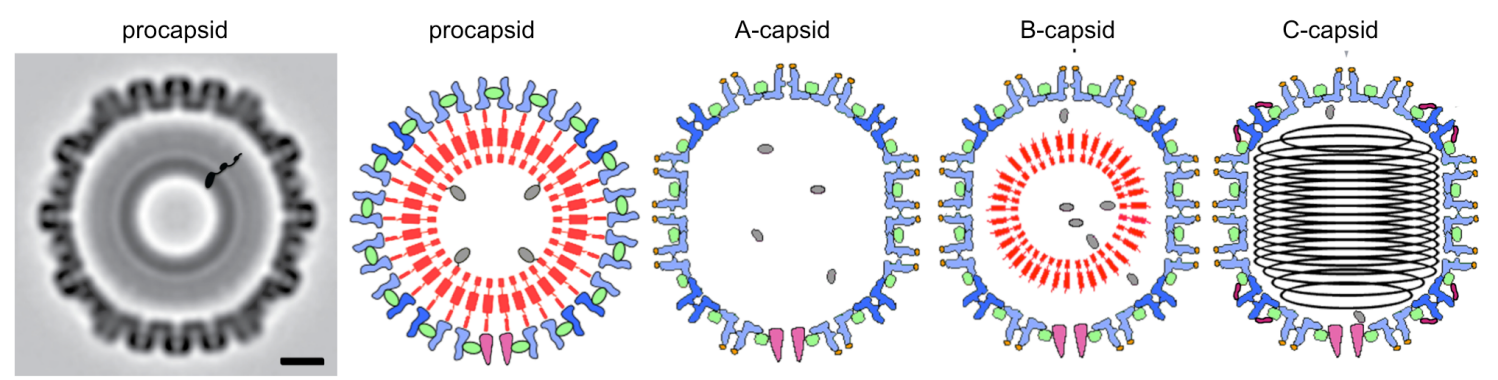

Figure 1. Central section of an averaged tomographic reconstruction of the procapsid, showing the full thickness of the inner shell and (schematically) the elongated conformation of preUL26.5. Also, schematic cross-sections of the four capsids [1]. Scale bar $=20 \mathrm{~nm}$.
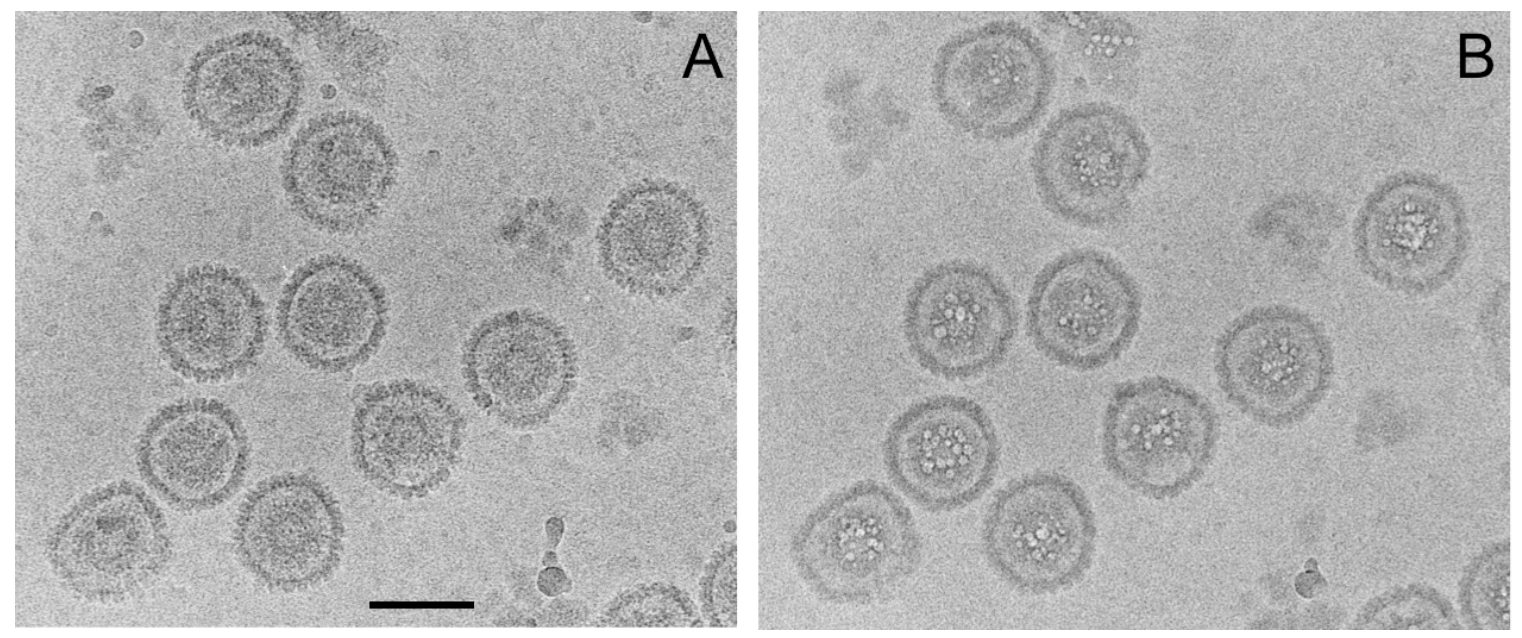

Figure 2. Cryo-micrographs of a field of procapsids, before bubbling (A, first exposure) and after initiating bubbling (B, ninth exposure). Scale bar $=100 \mathrm{~nm}$.
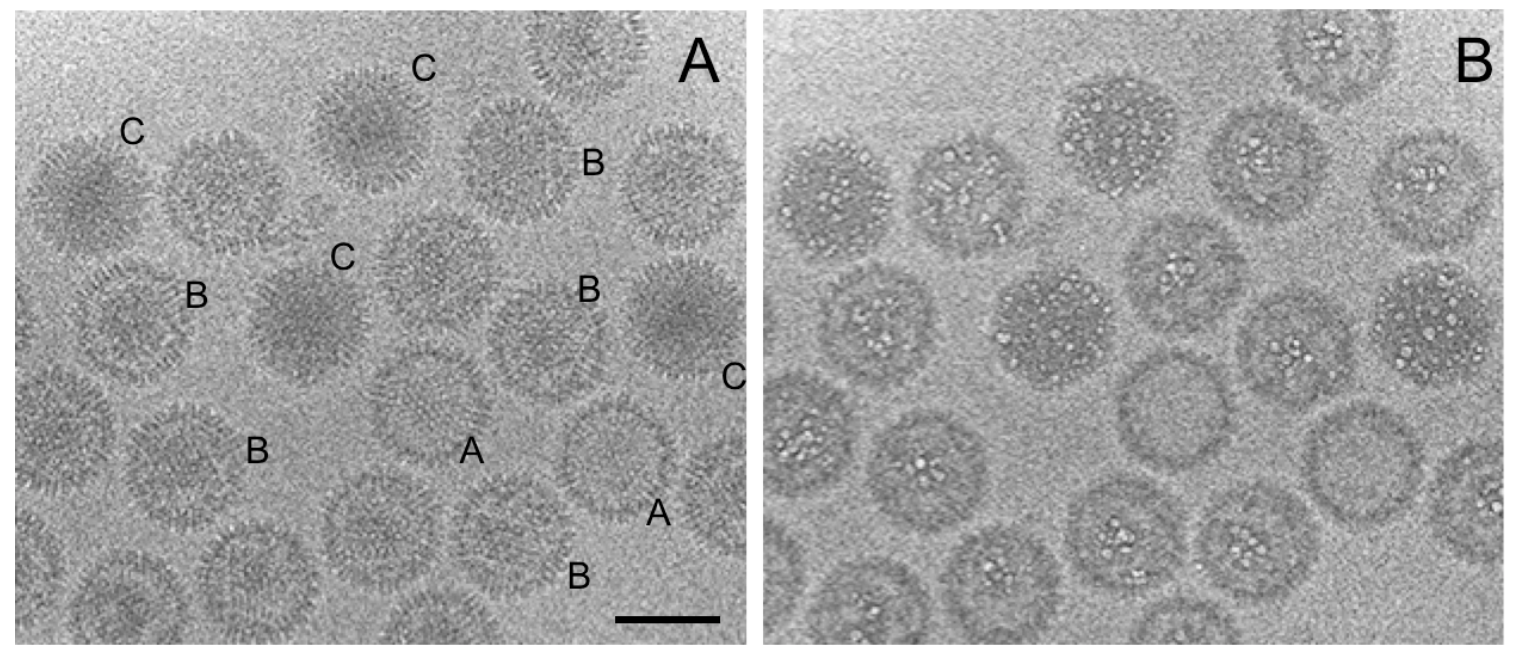

Figure 3. Cryo-micrographs of a mixed field of A- , B- and C-capsids, before bubbling (A, first exposure) and after initiating bubbling (B, thirteenth exposure). Scale bar $=100 \mathrm{~nm}$. 